\section{A 19-Year Old Female with Toxic Epidermal Necrolysis and Acute Pulmonary Failure was Successfully Treated with Extracorporeal Membrane Oxygenation: A Case Report and Review of the Literature}

\section{Roeleveld YWF, Cleffken BI, Dokter J, Oen IMMH and van der Vlies $\mathbf{C H}^{*}$}

Department of Surgery and Burns, Maasstad Hospital, Maasstadweg 21, 3079 Dz Rotterdam, Netherlands

\section{Case Report}

A 19-year old female was admitted to our burn centre in 2011 with a rapidly progressive TEN. 16 days before presentation she was prescribed with Pipemidic Acid (PPA) for a urinary tract infection, until 2 days before admission she had switched to nitrofurantoin.

On admission she had normotension with tachycardia of $100 \mathrm{bpm}$, tachypnea (16/minute), a maximal Glasgow Coma Scale and a temperature of $40.4^{\circ} \mathrm{C}$. She had blisters on her trunk, arms and head. Besides that she had petechiae on her legs. The affected skin involved $45 \%$ of her Total Body Surface Area (TBSA) (Figure 1). She was admitted to the ICU of our burn centre. The first day she was treated with amoxycillin and clavulanic acid and support was provided with rehydration and oxygen administration. Next day antibiotic treatment was changed to meropenem and gentamycin in response to bacteriological culture results: Haemolytic Streptococci type $B$ and Bacillus cereus. Prophylactic fluconazole and hydrocortisone were started.

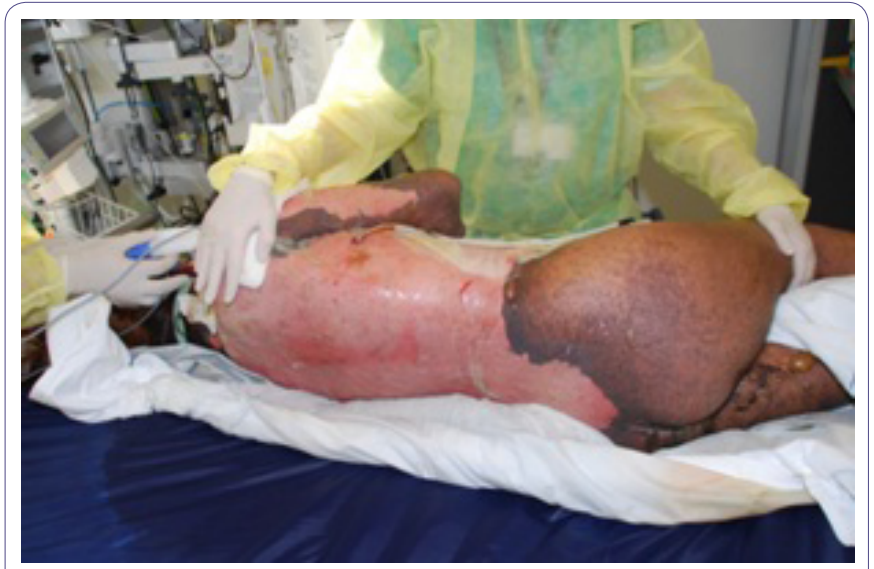

Figure 1: Large parts of her skin were affected.

One day after hospitalisation she developed a septic shock with high fever up to $41.2^{\circ} \mathrm{C}$. There was severe increase in surface area of the skin lesions (90\% TBSA). As a new finding mucous membranes of the eyes and the respiratory tract were affected. Serous secretions rapidly increased as a result of lesions in mucous linings. With anticipated troublesome intubation a primary tracheostomy was performed. Upon placement of the tracheostomy device roughly $400 \mathrm{ml}$ of serous secretion was evacuated.

On ventilator support the patient rapidly deteriorated with high pressure control ventilator settings $\left(\mathrm{FiO}_{2} 100 \%\right.$, positive end expiratory pressure $20 \mathrm{~mm} \mathrm{H}_{2} \mathrm{O}$, peak pressure $40 \mathrm{~mm} \mathrm{H}_{2} \mathrm{O}$ ), resulting in a $\mathrm{PaO}_{2}$ of $47 \mathrm{~mm} \mathrm{Hg}$. With Nitrogen ventilation initially there was some improvement $\left(\mathrm{FiO}_{2}\right.$ 90\%) in her respiratory failure. Pleural effusion and increasing interstitial abnormalities were visible on chest $\mathrm{X}$-ray (Figure 2). Pleural cavity drainage evacuated $250 \mathrm{ml}$ pleural effusion and with bronchial intermittent suctioning intrapulmonary effusion up to $150-300 \mathrm{ml}$ each 15 minutes was evacuated.

Being unable to support her with conventional mechanical ventilation, she was transferred by mobile intensive care unit to a 


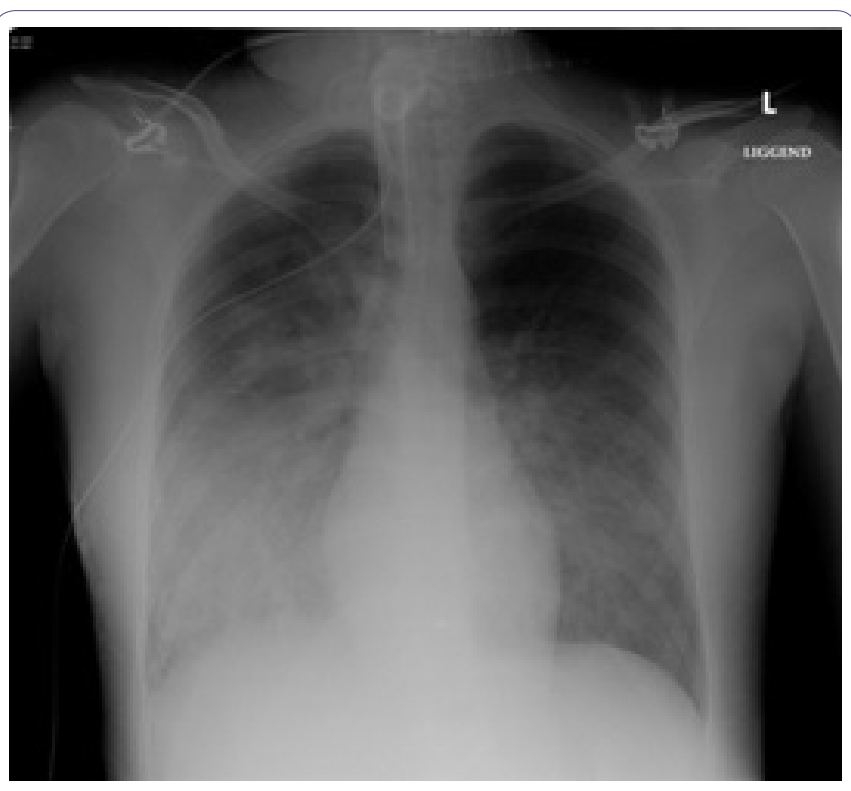

Figure 2: Chest X-ray showed pleural effusion and increasing interstitial abnormalities.

tertiary medical referral centre for ventilation with Extracorporeal Membrane Oxygenation (ECMO). The affected skin expanded to almost $100 \%$ TBSA. Prophylactic Zovirax was added to treatment. While on ECMO treatment, ventilator settings were lowered for peak pressure and $\mathrm{FiO}_{2}$ to minimize barotraumas. To prevent adhesion and collapse of alveoli PEEP was maintained at $20 \mathrm{~mm} \mathrm{H}_{2} \mathrm{O}$. Pulmonary conditions gradually improved, with diminishing secretions with bronchial evacuations. As a result patient was weaned of ECMO six days after transfer. She was transported back by mobile intensive care unit to our burn centre with regular ventilator settings.

During hospitalisation she developed a septic shock with Staphylococcus and Enterococcus bacteraemia as a result of skin lesion contamination. She was successfully treated with antibiotics. Her skin recovered with fast reepithelialization (Figures $3 \mathrm{~A}$ and $\mathrm{B}$ ). Twenty-one days after admission she was successfully weaned of the ventilator and her tracheostomy device was removed.

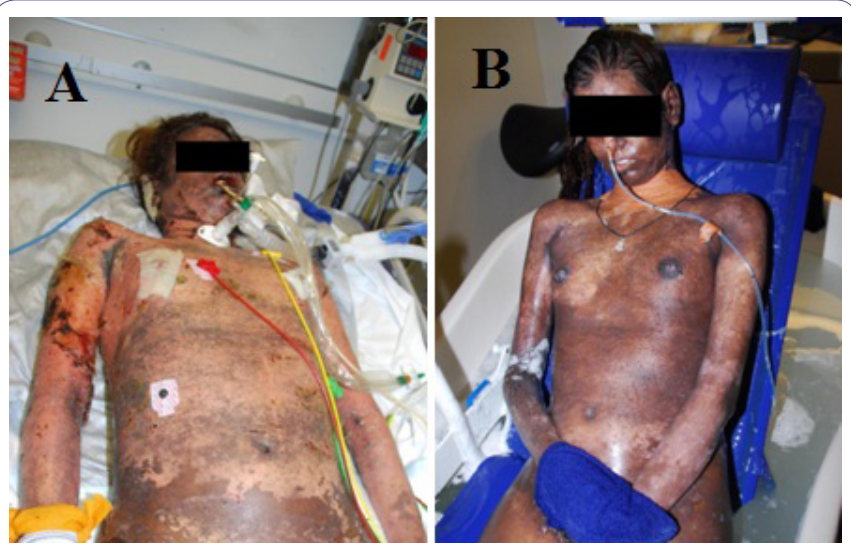

Figure 3: Re-epithalisation on day $18(\mathrm{~A})$ and day $32(\mathrm{~B})$ after admission.

Besides skin lesions and pulmonary problems, the patient's eyes were involved. Both conjunctivae and cornea of both eyes were affected. Her eyes were treated with corticosteroids from the first day of admission. The tendency to form symblepharon made the ophthalmologist decide to perform amnion membrane transplantation (Figure 4).

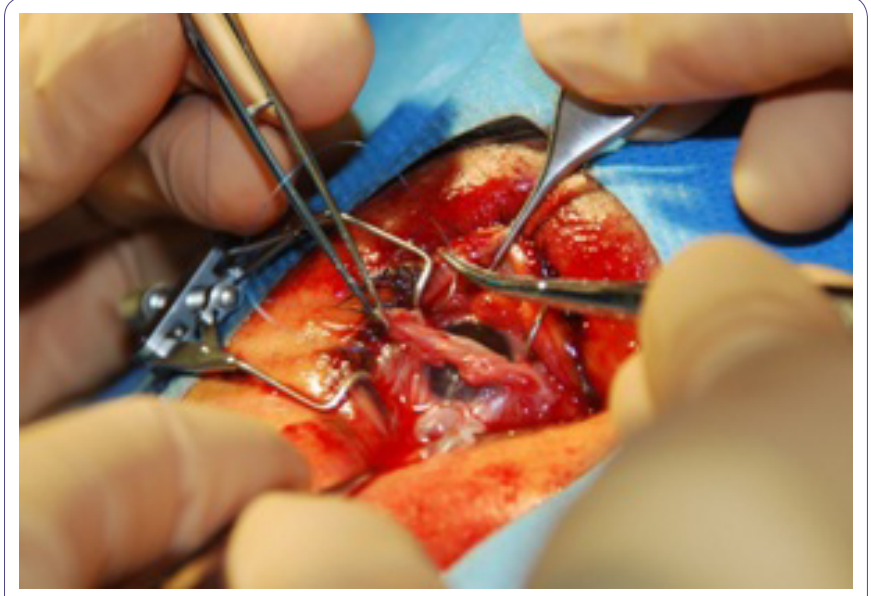

Figure 4: Amnion membrane transplantation.

Fourty-six days after admission she was transferred to rehabilitation centre. Her skin lesions spontaneously recovered, but post inflammatory hyper pigmentation remains. Her eyesight did not completely recover. Her vision is still reduced, caused by neovascularisation, symblepharon and corneal scarring.

\section{Discussion}

Toxic epidermal necrolysis, also known as Lyell's syndrome, was first described in 1956 by Alan Lyell. TEN is a rare condition with an incidence of approximately 1 to 2 cases per million per year [1,2]. TEN especially involves the skin and mucous membranes.

The histopathological feature is probably disruption of the epidermal-dermal junction, which results in hemorrhagic erosions, erythema and epidermal detachment, presenting as blisters and areas of denuded skin. The pathogenesis is probably immune mediated, but is not fully understood. T-lymphocytes have been identified to play a role in the initiation phase $[1,3]$.

In most cases, TEN are caused by medication (75\%) [3,4]. In the weeks before admission our patient used two different antibiotics; pipemidic acid and nitrofurantoin. Probably one of these drugs was the cause of TEN.

The treatment of TEN is based on supportive care and removal of the causative agent. Because a large part of the skin could be affected, patients are very sensitive to infections and dehydration. Treatment mainly consists of rehydration, antibiotic treatment if necessary and wound dressings. This combination of care can ideally be given in a burn centre. With larger percentages of TBSA affected, sometimes admission to an intensive care unit is necessary.

In TEN patients mucous linings like the eyes, the buccal mucosa, the respiratory system, the gastro-intestinal tract and the urogenital tract can be affected [5]. In over $50 \%$ of all patients diagnosed with TEN, treated at a burn centre, the eyes are affected [6-8]. In our patient, the eyes and the respiratory tract were severely affected.

Her eyes were initially treated with eye drops and ointment: trimethoprim/polymyxin B sulfate, ofloxacine and corticosteroids. The tendency to form symblepharon made the ophthalmologist decide to perform an Amnion Membrane Transplantation (AMT). The most common treatment for ocular involvement is topical antibiotics, topical steroids and lubricants [9]. In AMT the amnion 
Citation: Roeleveld YWF, Cleffken BI, Dokter J, Oen IMMH, van der Vlies CH (2015) A 19-Year Old Female with Toxic Epidermal Necrolysis and Acute Pulmonary Failure was Successfully Treated with Extracorporeal Membrane Oxygenation: A Case Report and Review of the Literature. J Clin Dermatol Ther 2: 010.

- Page 3 of $3 \cdot$

membrane is sutured to the eyelids to cover the entire ocular surface as a temporary biological bandage. AMT in patients with TEN was first reported by John et al., in 2002 [10]. Since then AMT in patients with TEN was described in some cases. Recent literature suggests that AMT suppresses inflammation, prevents ulcer formation and promotes healing, and as a consequence prevents sight-threatening sequelae $[11,12]$.

Our patient developed also pulmonary complications with the need of ventilation with ECMO. Conventional mechanical ventilation was insufficient to support her. Bronchial suctioning was performed every 15 minutes, necessitated by desaturations, evacuating up to $300 \mathrm{ml}$ each time, with end expiratory pressures of $20 \mathrm{~mm} \mathrm{H}_{2} \mathrm{O}$. She was transferred to a tertiary medical centre for ventilation with ECMO. To our knowledge, in the literature only two case reports about a TEN-patient treated with ECMO were described $[13,14]$.

The comparison between these cases is the development of Acute Respiratory Distress Syndrome (ARDS) with occurrence of large volumes of broncho-alveolar secretions due to the mucous lesions in her respiratory system. Other causes of pulmonary complications in TEN patients could be a community-acquired pneumoniae, a nosocomial mechanical ventilation infection or a combination of these.

Because full recovery is typical in TEN patients, these patients are ideal candidates for ECMO, when severe pulmonary dysfunctions develop as demonstrated in these cases. $[13,14]$ ARDS is the most common cause for pulmonary complications in TEN patients.

As showed in this case, TEN can be a life-threatening condition. The mortality rate of TEN is $25-35 \%$ [1]. Sepsis is the most common cause of death [3]. The prognosis for TEN can be predicted on basis of the SCORTEN score (Table 1). This is a prognostic scoring system especially for patients with TEN, using 7 determinants observed within the first 24 hours after admission [15]. Our patient had a SCORTEN score of three (heart rate $\geq 120 / \mathrm{min}$ and TBSA involved at day $1>10 \%$, Serum bicarbonate $<20 \mathrm{mmol} / \mathrm{L}$ ), which corresponds to a predicted mortality of $35.3 \%$. On the first day of hospitalisation, her prognosis was relatively good. On the second day her pulmonary conditions deteriorated and the affected TBSA expanded to almost $100 \%$. She developed the need for ventilation support and with ongoing respiratory insufficiency the need for ECMO. Clearly her clinical status was with a poor prognosis, although her SCORTEN score was still three.

\begin{tabular}{|c|c|c|}
\hline Items Presence $=\mathbf{1}, \mathbf{A b s e n c e}=\mathbf{0}$ & Score & Predicted Mortality \\
\hline Age $\geq 40$ years & $0-1$ & $3.2 \%$ \\
\hline Malignancy & 2 & $12.1 \%$ \\
\hline Heart rate $\geq 120 / \mathrm{min}$ & 3 & $35.3 \%$ \\
\hline TBSA involved at day $1>10 \%$ & 4 & $58.3 \%$ \\
\hline Serum urea $>10 \mathrm{mmol} / \mathrm{L}$ & $>5$ & $90.0 \%$ \\
\hline Serum glucose $>14 \mathrm{mmol} / \mathrm{L}$ & \multicolumn{2}{|}{} \\
\hline Serum bicarbonate $<20 \mathrm{mmol} / \mathrm{L}$ & \multicolumn{2}{|}{} \\
\hline
\end{tabular}

Table 1: SCORTEN score.

\section{Conclusion}

TEN with nearly $100 \%$ TBSA involvement can be treated with full recovery of the skin. In a patient with pulmonary complications (ARDS) secondary to TEN, ECMO should be considered as a rescue therapy. Furthermore, amnion membrane transplantation for ocular involvement could be an option for treatment. Our patient recovered completely with only sequelae to eyes and hyperpigmentation of the skin.

\section{References}

1. Harr T, French LE (2010) Toxic epidermal necrolysis and Stevens-Johnson syndrome. Orphanet J Rare Dis 5: 39.

2. Lyell $A$ (1956) Toxic epidermal necrolysis: an eruption resembling scalding of the skin. Br J Dermatolog 68: 355-361.

3. Mockenhaupt M (2011) The current understanding of Stevens-Johnson syndrome and toxic epidermal necrolysis. Expert Rev Clin Immunol 7: 803-813.

4. Roujeau JC, Kelly JP, Naldi L, Rzany B, Stern RS, et al. (1995) Medication use and the risk of Stevens-Johnson syndrome or toxic epidermal necrolysis. N Engl J Med 333: 1600-1607.

5. Revuz J, Penso D, Roujeau JC, Guillaume JC, Payne CR, et al. (1987) Toxic epidermal necrolysis. Clinical findings and prognosis factors in 87 patients. Arch Dermatol 123: 1160-1165.

6. Palmieri TL, Greenhalgh DG, Saffle JR, Spence RJ, Peck MD, et al. (2002) A multicenter review of toxic epidermal necrolysis treated in US burn centers at the end of the twentieth century. J Burn Care Rehabil 23: 87-96.

7. Gerdts B, Vloemans AF, Kreis RW (2007) Toxic epidermal necrolysis: 15 years' experience in a Dutch burns centre. J Eur Acad Dermatol Venereol 21: 781-788.

8. Khoo AK, Foo CL (1996) Toxic epidermal necrolysis in a burns centre: a 6-year review. Burns 22: 275-278.

9. Chang YS, Huang FC, Tseng SH, Hsu CK, Ho CL, et al. (2007) Erythema multiforme, Stevens-Johnson syndrome, and toxic epidermal necrolysis: acute ocular manifestations, causes, and management. Cornea 26: 123-129.

10. John T, Foulks GN, John ME, Cheng K, Hu D (2002) Amniotic membrane in the surgical management of acute toxic epidermal necrolysis. Ophthalmology 109: 351-360.

11. Shay E, Kheirkhah A, Liang L, Sheha H, Gregory DG, et al. (2009) Amniotic membrane transplantation as a new therapy for the acute ocular manifestations of Stevens-Johnson syndrome and toxic epidermal necrolysis. Surv Ophthalmol 54: 686-696.

12. Gregory DG (2011) Treatment of acute Stevens-Johnson syndrome and toxic epidermal necrolysis using amniotic membrane: a review of 10 consecutive cases. Ophthalmology 118: 908-914.

13. Ryan DP, Doody DP (1992) Treatment of acute pulmonary failure with extracorporeal support: $100 \%$ survival in a pediatric population. J Pediatr Surg 27: $1111-1116$

14. Sine CR, Chung KK, Pamplin JC, Batchinsky Al, Hull JE, et al. (2014) Extracorporeal membrane oxygenation in a patient with refractory Acute Respiratory Distress Syndrome secondary to toxic epidermal necrolysis. J Burn Care Res 35: 428-430.

15. Bastiju-Garin S, Fouchard N, Bertocchi M, Roujeau JC, Revuz J, et al. (2000) SCORTEN: a severity-of-illness score for toxic epidermal necrolysis. J Invest Dermatol 115: 149-153. 